\title{
Signature of the black hole phase transition in quasinormal modes
}

\author{
Xi He, Bin Wang \\ Department of Physics, Fudan University, 200433 Shanghai \\ Rong-Gen Cai \\ Institute of Theoretical Physics, Chinese Academy of Sciences, P.O. Box 2735, 100190 Beijing \\ Chi-Yong Lin \\ Department of Physics, National Dong Hwa University, Shoufeng, 974 Hualien
}

\begin{abstract}
We study the perturbation of the scalar field interacting with the Maxwell field in the background of d-dimensional charged AdS black hole and AdS soliton. Different from the single classical field perturbation, which always has the decay mode in the black hole background, we observe the possible growing mode when the perturbation of the scalar field strongly couples to the Maxwell field. Our results disclose the signature of how the phase transition happens when the interaction among classical fields is strong. The sudden change of the perturbation to growing mode is also observed in the AdS soliton with electric potential. However in the magnetic charged AdS soliton background, we observe the consistent perturbation behavior when the interaction between scalar field and Maxwell field is considered. This implies that for the magnetic charged AdS soliton configuration, unlike the situation with electric potential, there is no scalar field condensation which causes the phase change.
\end{abstract}

PACS numbers: 04.70.Dy, 95.30.Sf, 97.60.Lf 


\section{INTRODUCTION}

The quasinormal mode (QNM) of black holes describes the damped oscillations under perturbations in the surrounding geometry of a black hole with frequencies and damping times of the oscillations entirely fixed by the black hole parameters. The QNM is considered as the characteristic sound of black holes and is expected to be detected through gravitational wave observations [1, 2]. In addition to its potential astrophysical interest to directly identify the black hole existence, the black hole QNM has been argued recently as a useful testing ground for fundamental physics. It is widely believed that the study of QNM can help us get deeper understandings of the AdS/CFT [3-7], dS/CFT [8] correspondences [9], loop quantum gravity [10] and present us the imprint of the extra dimensions [11, 12]. Furthermore it has been argued that the QNM can reflect the black hole phase transition [13-15].

In most studies of the QNM, the wave dynamics of a single classical field propagating outside the black hole is taken into account. For the stable black hole backgrounds, the perturbation of a single classical field will die out finally due to the black hole absorption. Recently more interest has been focused on the strongly coupled theory by considering the Einstein-Maxwell field interacting with a charged scalar field with a minimal Lagrangian

$$
\mathcal{L}=-\frac{1}{4} F_{\mu \nu}^{2}-\left|\partial_{\mu} \Psi-i q A_{\mu} \Psi\right|^{2}-m^{2}|\Psi|^{2} .
$$

In the light of AdS/CFT correspondence, it was realized that a condensate of the charged scalar field is formed through its coupling to a Maxwell field of the background [16]. Along this line, there have been a lot of investigations concerning the application of AdS/CFT correspondence to condensed matter physics by considering interactions among classical fields [17-23]. See Refs. [24, 25] for reviews. It was disclosed that there is a second order transition, with mean field theory exponents, between a non-superconducting state at high temperatures, where all the charge is in the normal component, and a superconducting state at low temperatures. Similar transition occurs for black hole geometries based on gravity coupled to the Abelian Higgs model [26, 27]. It is of great interest to ask whether the QNM can be an effective probe of this phase transition. This is the motivation of the present work.

In [28], the marginally stable linearized perturbation around a charged black hole background in AdS space that breaks the $U(1)$ symmetry has been discussed. In this work we want to establish the more range of behaviors that this mechanism exhibits by studying the QNM behavior. In [29], the QNM at strong coupling was computed from the poles of retarded Green's function in the black hole background. In our investigation 
we will start from the bulk equations and directly study the wave dynamics outside black holes. By this way we can get the objective picture of how the perturbation of the scalar field evolves if it is coupled to a Maxwell field. By comparing our result with that obtained in [29], we can have further insight into the holographic correspondence.

Besides the black hole configuration, in our study we will also investigate the AdS soliton configuration which has lower energy than the AdS space in the Poincare coordinates, but has the same boundary topology as the Ricci flat black hole and the AdS space in the Poincare coordinates [30]. It was found that there is a Hawking-Page phase transition between the Ricci flat AdS black hole and the AdS soliton [31, 32] and this phase transition was observed in the QNM spectrum [14]. Recently it was argued that a phase transition between an AdS soliton configuration and a superconducting phase will appear if the chemical potential is changed [33]. We are going to investigate whether this phenomenon can be reflected in the QNM behavior.

The organization of the paper is as follows. In Sec. II we will present the charged black hole backgrounds of interest and discuss associated wave dynamics. In Sec. III we will investigate the QNMs of AdS soliton. Conclusions and discussions will be presented in Sec. IV.

\section{THE REISSNER-NORDSTRÖM (RN) BLACK HOLES IN ADS SPACES}

The electrically charged black hole in $d$-dimensional AdS space is described by the metric

$$
d s^{2}=-f d t^{2}+\frac{1}{f} d r^{2}+r^{2} h_{i j} d x^{i} d x^{j}
$$

where

$$
f=k-\frac{2 M}{r^{d-3}}+\frac{Q^{2}}{4 r^{2 d-6}}+\frac{r^{2}}{L^{2}}
$$

and $M$ and $Q$ are referred to black hole mass and charge. $L$ here is the AdS radius. The black hole horizon $r_{+}$ can be obtained from $f(r)=0 . h_{i j} d x^{i} d x^{j}$ is the line element for flat $(k=0)$, spherical $(k>0)$ or hyperbolic $(k<0)(d-2)$-plane. The Hawking temperature defined as $T=f^{\prime}\left(r_{+}\right) / 4 \pi$ is

$$
T=\frac{r_{+}^{-2 d-1}}{16 \pi L^{2}}\left[4(d-1) r_{+}^{2 d+2}-(d-3) L^{2}\left(Q^{2} r_{+}^{6}-4 k r_{+}^{2 d}\right)\right] .
$$

The gauge field ansatz is

$$
A=\Phi(r) d t
$$


We can find a simple solution to the equations of motion from the Lagrangian (1) together with the EinsteinHilbert Lagrangian plus a negative cosmological constant, which is in the form

$$
\Phi=\sqrt{\frac{d-2}{2(d-3)}}\left(\frac{Q}{r^{d-3}}-\frac{Q}{r_{+}^{d-3}}\right), \quad \Psi=0 .
$$

Considering the scalar field $\Psi$ perturbing around the $\mathrm{RN}-\mathrm{AdS}_{d}$ black hole, we have the wave equation of the charged scalar field directly from the Lagrangian (10)

$$
\left[\frac{1}{\sqrt{-g}} \frac{\partial}{\partial x^{\mu}}\left(\sqrt{-g} g^{\mu \nu} \frac{\partial}{\partial x^{\nu}}\right)-m_{e f f}^{2}\right] \Psi=0
$$

where the effective mass of the scalar field is of the form

$$
m_{\text {eff }}^{2}=m^{2}+g^{t t} q^{2} \Phi^{2}
$$

Since $g^{t t}$ is negative outside the horizon and diverges to $-\infty$ near the horizon, it seems possible that $m_{\text {eff }}^{2}$ should become negative which will directly lead to the unstable perturbation in the following discussion. The radial part of the equation (7) can be separated by setting $\Psi=e^{-i \omega t} r^{\frac{2-d}{2}} R(r) S\left(x_{i}\right)$. Defining the tortoise coordinate $d r_{*}=d r / f(r)$, the radial wave equation can be expressed as

$$
\frac{d^{2} R(r)}{d r_{*}^{2}}+\left[\omega^{2}-V(r)\right] R(r)=0
$$

with the effective potential

$$
V(r)=\frac{(d-2)(d-4)}{4 r^{2}} f^{2}+\frac{\lambda^{2}}{r^{2}} f+m_{e f f}^{2} f+\frac{d-2}{2 r} f f^{\prime},
$$

and the separation constant $\lambda^{2}=l(l+d-3),(l=0,1,2 \ldots)$. Throughout this paper we will only consider $l=0$ for simplicity.

Choosing scaling symmetry adopted in [28], we can fix $r_{+}=Q=1$ and change the value of $L$ to vary the temperature of the black hole. We follow [28] to fix $m^{2} L^{2}$ and $q L$ as we vary $L$. For selecting $m^{2} L^{2}=$ 4, $q L=10$, the effective potential for the $\mathrm{RN}_{-} \mathrm{AdS}_{4}$ black hole background is shown in the left of Fig 1 The negative potential well appears due to the negative value of the effective mass term in Eq.(8). When $r \rightarrow \infty$, the potential approaches the positive infinity. With the increase of the AdS radius, the temperature of the black hole becomes smaller and we see that the negative potential well becomes wider. The behavior of the effective potential changes drastically when $m^{2}<0$. Choosing $m^{2} L^{2}=-2, q L=3$, we show the effective potential in the right of Fig 1 We see that the potential is almost negative except a small positive barrier near horizon for small $L$. When $r \rightarrow \infty$, the potential approaches $V(r) \rightarrow-9 / L^{2}$. For other dimensions, the 

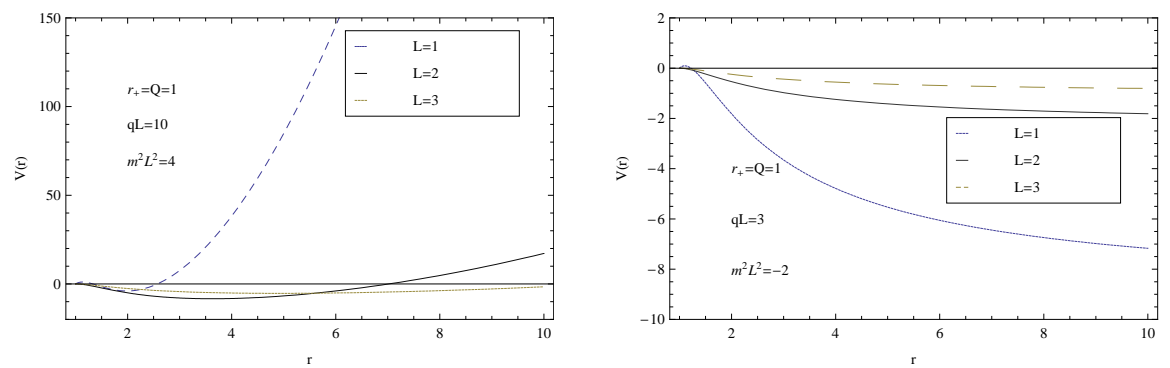

FIG. 1: The behavior of the effective potential. The left panel is for selecting $q L=10, m^{2} L^{2}=4$, and the right panel is for $q L=3, m^{2} L^{2}=-2$. In both cases we set $r_{+}=Q=1$.

potential behaviors are similar. It was argued in [8] that the behavior of the effective potential influences a lot on the wave dynamics. In the following we are going to examine the evolution of the wave propagation of the perturbed field.

Using the new variables $v=t-r_{*}, u=t+r_{*}$, we can rewrite Eq.(9) into

$$
\frac{d^{2} R}{d u d v}+\frac{V(r)}{4} R=0
$$

This equation can be discretized as

$$
R_{N}=R_{E}+R_{W}-R_{S}+\Delta u \triangle v V\left(\frac{v_{N}+v_{W}-u_{N}-u_{E}}{4}\right) \frac{R_{W}+R_{E}}{8}+O\left(\epsilon^{2}\right)
$$

where the points $N, S, E$, and $W$ form a null rectangle with relative positions as: $N:(u+\triangle u, v+\triangle v)$, $W:(u+\triangle u, v), E:(u, v+\triangle v)$ and $S:(u, v)$. The parameter $\epsilon$ is an overall grid scale factor, so that $\triangle u \sim \triangle v \sim \epsilon$.

In presenting the numerical results, we fix $r_{+}=Q=1$ and vary the AdS radius $L$ which corresponds to the change of the black hole temperature. The left panel of Fig.2 shows the objective picture of the evolution of perturbation for choosing $m^{2} L^{2}=4, q L=10$ in the 4-dimensional flat black hole background. We observe that with the increase of $L$, the temperature of the black hole drops, the perturbation oscillates less and lasts longer. When $L_{*}=1.11$, the perturbation does not oscillate and stops decaying. This value of $L$ coincides with the marginally stable mode observed in [28]. When $L>L_{*}$, the perturbation outside the black hole keeps growing. The blow up of the perturbation indicates that when the black hole temperature is under some critical value, the original black hole configuration is no longer stable. When this occurs, the scalar will start to condensate and address to the black hole configuration, which was described by the superconducting state in the language of AdS/CFT correspondence 24].

Considering the case for $m^{2} L^{2}=4, q L=10$, the potential approaches positive infinity, which puts $\Psi=0$ at the boundary. We can use the Horowitz-Hubeny method [3], which has been used extensively in previous 

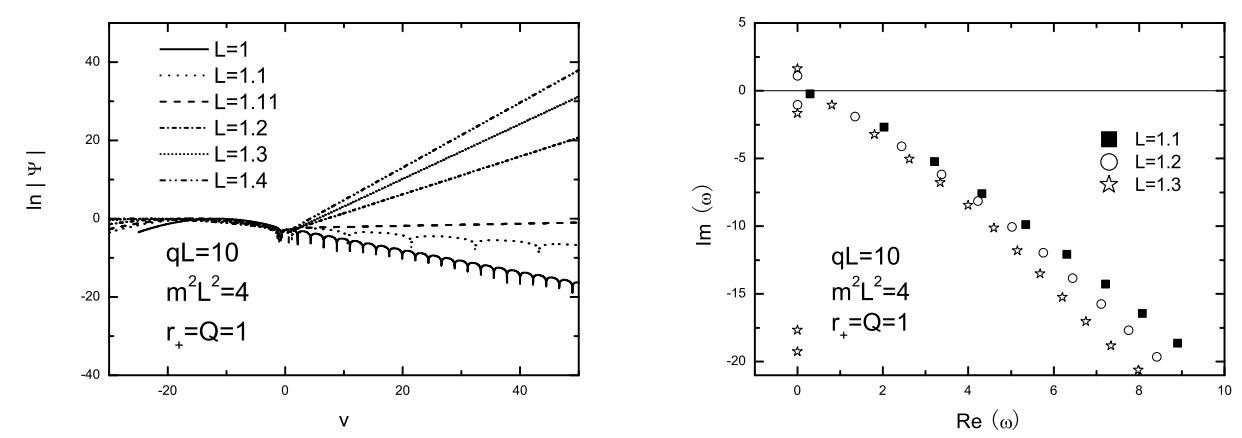

FIG. 2: The QNM of charged scalar field around flat RN-AdS 4 black hole when we take $q L=10, m^{2} L^{2}=4$.
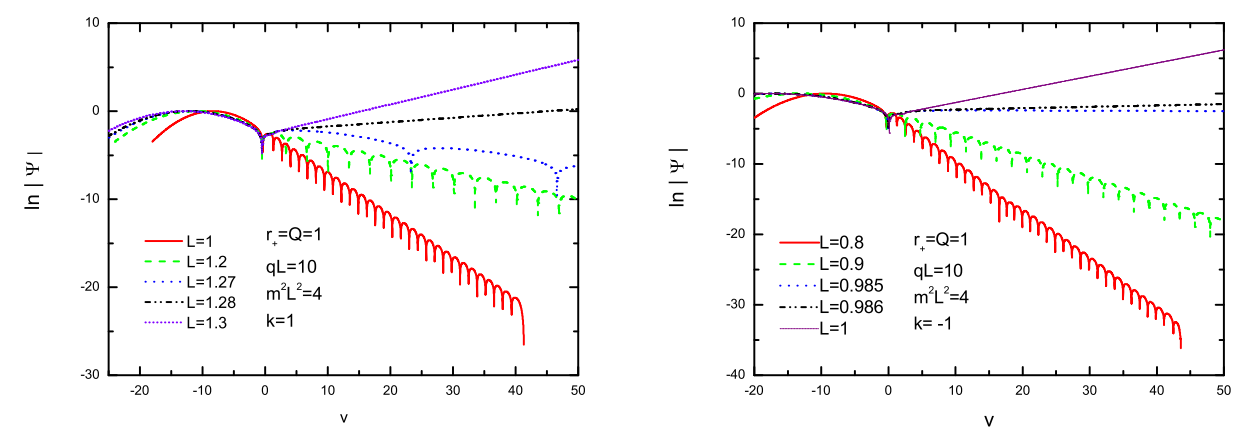

FIG. 3: The QNM of charged scalar field around the spherical RN- $A d S_{4}$ black hole (left) and hyperbolic RN- $A d S_{4}$ black hole (right) for setting $q L=10, m^{2} L^{2}=4$.
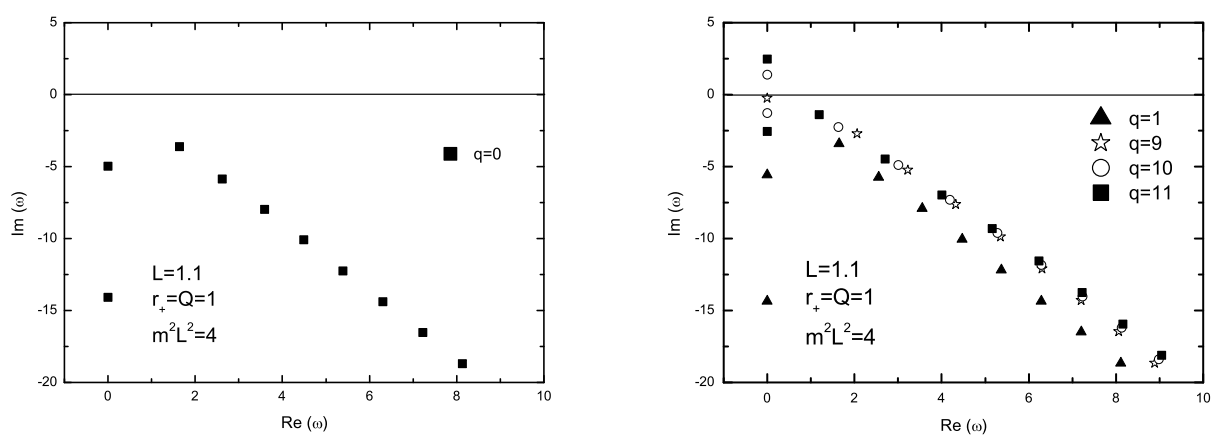

FIG. 4: The influence of the quasinormal frequency in the background of the flat $\mathrm{RN}-\mathrm{AdS}_{4}$ black hole due to the value of $q$. Here we set $L=1.1, m^{2} L^{2}=4$. The left panel is for $q=0$ and the right one is for the increase of $q$. 

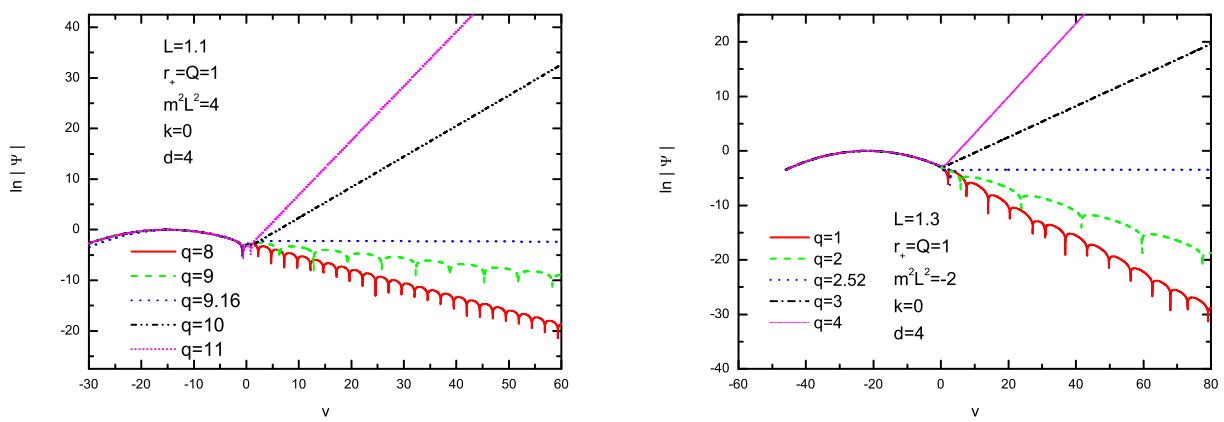

FIG. 5: The objective picture of the dependence of the QNM of flat RN-AdS 4 black hole on values of $q$. The left panel is for choosing $m^{2} L^{2}=4, L=1.1$ and we find $q_{*}=9.16$, while the right panel is for selecting $m^{2} L^{2}=-2, L=1.3$ and we have $q_{*}=2.52$.
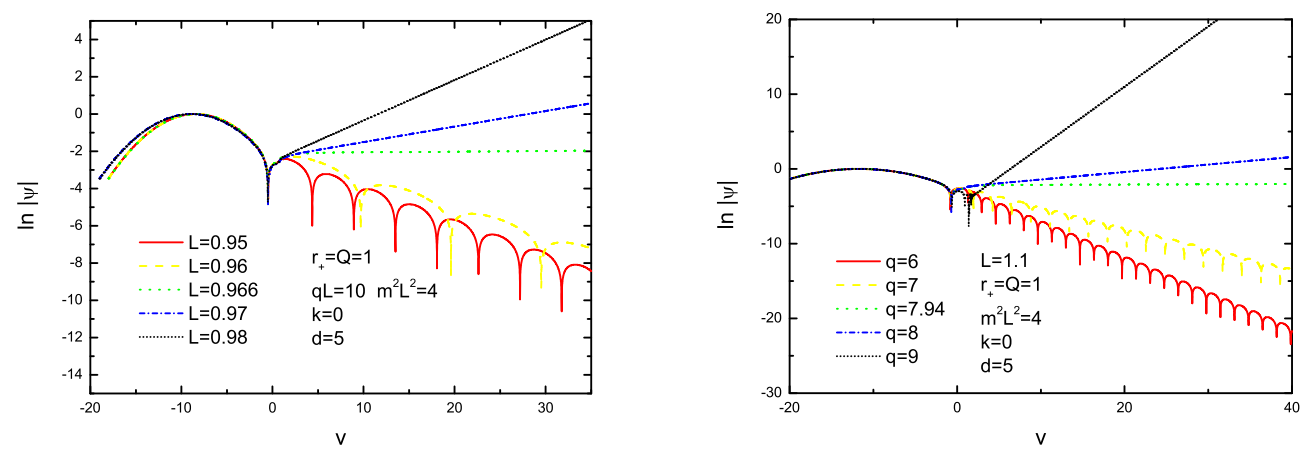

FIG. 6: The objective picture of the QNM for RN-AdS 5 black hole. The left panel is for selecting $q L=10, m^{2} L^{2}=4$ and we get the critical AdS radius $L_{*}=0.966$. The right panel shows the result changing with $q$ by fixing $L=1.1$, $m^{2} L^{2}=4$ and we get the critical value of the charge of the scalar field to break the stability at $q_{*}=7.94$.

papers $[4,5]$ to directly calculate the quasinormal frequencies of the perturbation. The result is presented in the right panel of Fig.2. As the increase of $L$, the black hole temperature drops, the topmost mode will move into the upper half frequency plane, indicating the growing mode and the instability of the black hole configuration. The critical value $L_{*}=1.11$ agrees with that observed in the objective picture. The behavior of the quasinormal frequency we obtained from solving the bulk equation is in consistent with that from poles in the retarded Green's function [29].

In Fig.3 we also present the result of wave dynamics for 4-dimensional spherical and hyperbolic black hole backgrounds. Choosing the same values of $m^{2} L^{2}, q L$ as those of the flat case, we see that in the spherical black hole background, the black hole starts to be unstable due to the perturbation at bigger value of the AdS radius $L_{*}$, while for the hyperbolic case, the black hole begins unstable at smaller $L_{*}$ than that of the 
flat case. Inserting these $L_{*}$ into the temperature expression, we observe that the critical temperature to break the background stability satisfies $T_{*(k=1)}>T_{*(k=0)}>T_{*(k=-1)}$. This shows that the topology of the spacetime has the influence on evolution of the perturbation. The spherical background is the easiest to be destroyed due to the perturbation. This phenomenon provides the motivation to examine the spacial topology influence on the condensation. Recently it was argued that the negative curvature topology can make the superconductor gapless and give a geometrical mechanism of conductivity [21]. More studies in this direction is called for.

The growing mode was caused by the interaction between scalar field and the Maxwell field, which has never been observed in the perturbation of a single classical field in the black hole background [4 6]. The process of the blow up (or the scalar condensation) can be understood as arising in part from the temperature of the black hole, but also as arising from the other two factors which lead to the negative effective mass of the scalar field, the charge $q$ and the mass $m$ of the scalar field. To see the influence of these two factors, we fix the value of the black hole background, $r_{+}, Q$ and $L$. If $q$ is big, the electric field outside the horizon is big. For the same mass of the scalar field, $m_{\text {eff }}^{2}$ will become negative provided that $q$ is big enough, this can be seen from (8). The influence of $q$ on the evolution of the perturbation is shown in Fig.4. When $q=0$, there is no coupling between the scalar field and the Maxwell field, the perturbation always decays. However with the increase of the $q$, it is possible for $m_{\text {eff }}^{2}$ to become negative. The topmost mode moves upward with the increase of $q$ and when $q$ is over some critical value $q_{*}$ the topmost mode will move into the upper half of the frequency plane indicating the instability of the background. The objective picture with the increase of $q$ is also shown in Fig.5. For smaller mass of the scalar field, it is easier for $m_{\text {eff }}^{2}$ to be negative outside the horizon and to produce an unstable mode in $\Psi$, this can happen even for the smaller value of $q_{*}$, which is shown in the right panel of Fig.5.

In Fig.6, we also exhibit the result of the perturbation for 5-dimensional flat black hole background. Comparing with that of the 4-dimensional situation (shown in Fig.2), the blow up of the perturbation can appear at smaller values of $L_{*}$ and $q_{*}$. For fixing $r_{+}=Q=1$, inserting the values of $L_{*}$ into the temperature expression, we have $T_{* 4 D}>T_{* 5 D}$. This shows that the instability of the 4-dimensional RN AdS black hole can happen at higher temperature than that in 5-dimensions. However when we fix the same AdS radius in 4-dimensions and 5-dimensions, we observe that the critical value of the charge of the scalar field to break the stability is smaller for the 5 -dimensional case. This implies that for the same black hole parameters $\left(r_{+}, Q, L\right)$, the stability of the black hole spacetime can be broken easier in the high dimensions. This result supports the 
finding that the scalar hair can be formed easier in the higher dimensional background [22].

\section{THE ADS SOLITON BACKGROUND}

In this section we will study the perturbation of the scalar field coupled to Maxwell field in the background of AdS soliton. Recently the signature of the Hawking-Page phase transition between the Ricci flat AdS black hole and the AdS soliton [30 32] was observed in the QNM spectrum of the scalar perturbation in [14]. It was argued that there is a phase transition in the AdS soliton configuration and the superconducting phase will appear dual to an AdS soliton [33]. It is of interest to study the evolution of the perturbation in the charged AdS soliton background and get more understanding on this phase transition.

By analytically continuing the Ricci flat black hole one obtains the AdS soliton [30, 31]

$$
d s^{2}=-r^{2} d t_{s}^{2}+\frac{1}{f_{s}} d r^{2}+f_{s} d \phi_{s}^{2}+r^{2} h_{i j} d x^{i} d x^{j}
$$

To satisfy the Einstein equation the function $f_{s}$ is in the same form as Eq.(3) but with a negative sign before $Q^{2}$. We have $k=0$ and $r_{s}$ replacing $r_{+}, \phi_{s}$ is identified as the period $\beta_{s}=4 \pi / f^{\prime}\left(r_{s}\right)$ and $h_{i j} d x^{i} d x^{j}$ denotes a $(d-3)$-dimensional Euclidean space. Now the the gauge field is transformed into

$$
A=\Phi(r) d \phi_{s}
$$

This corresponds to a magnetic field $F_{\phi_{s} r}=d \Phi(r) / d r$, while Eq. (5) leads to a radial electric field.

With the variable separation $\Psi(r)=e^{-i \omega t} r^{\frac{2-d}{2}} R(r) S(\theta) Y\left(\phi_{s}\right)$, we obtain

$$
\frac{d^{2} R(r)}{d r^{2}}+\frac{f_{s}^{\prime}}{f_{s}} \frac{d R(r)}{d r}+\left[-\frac{(d-2)(d-4)}{4 r^{2}}-\frac{d-2}{2 r} \frac{f^{\prime}}{f}+\frac{\omega^{2}}{f r^{2}}-\frac{m_{e f f}^{2}}{f}-\frac{\lambda^{2}}{f r^{2}}-\frac{k_{s}^{2}}{f^{2}}\right] R(r)=0
$$

where the effective mass is

$$
m_{e f f}^{2}=m^{2}+g^{\phi \phi} q^{2} \Phi^{2}, \quad \Phi=\sqrt{\frac{d-2}{2(d-3)}}\left(\frac{Q}{r_{s}}-\frac{Q}{r}+A\right) .
$$

$A$ here is a constant. $\lambda^{2}$ and $k_{s}^{2}=\frac{2 \pi \tilde{m}}{\eta_{s}}(\tilde{m}=0,1,2 \ldots)$ are the eigenvalues of

$$
\begin{aligned}
& \frac{d^{2} S(\theta)}{d \theta^{2}}+\lambda^{2} S(\theta)=0, \\
& \frac{d^{2} Y\left(\phi_{s}\right)}{d \phi_{s}^{2}}+k_{s}^{2} Y\left(\phi_{s}\right)=0 .
\end{aligned}
$$

We observed that the negative effective mass term plays an important role in producing growing modes in the RN-AdS black hole. However in the charged AdS soliton case, due to the coordinate transformation, $g^{\phi \phi}$ substitutes $g^{t t}$ in the second term of the effective mass. Since $g^{\phi \phi}$ is always positive, the effective mass will 
TABLE I: Normal Modes for magnetic charged $\mathrm{AdS}_{4}$ soliton, we set $r_{+}=Q=L=1, q=10$. We show the results of the first three modes with overtone numbers $n=0,1,2$ from the top to the bottom.

\begin{tabular}{|c|c|c|c|}
\hline$m^{2}=2$ & $m^{2}=1$ & $m^{2}=0$ & $m^{2}=-1$ \\
\hline 4.0612862 & 3.8935134 & 3.7161216 & 3.5271927 \\
6.7874836 & 6.6222613 & 6.4452027 & 6.2519047 \\
9.0955200 & 8.9047114 & 8.6926610 & 8.4464558 \\
\hline
\end{tabular}

TABLE II: Normal Modes for magnetic charged $\mathrm{AdS}_{4}$ soliton, we set $r_{+}=Q=L=1, m^{2}=0$. We show the results of the first three modes with overtone numbers $n=0,1,2$ from the top to the bottom.

\begin{tabular}{|c|c|c|c|}
\hline$q=10$ & $q=5$ & $q=1$ & $q=0$ \\
\hline 3.7161216 & 3.0973695 & 2.7779244 & 2.7621022 \\
6.4452027 & 5.4999791 & 5.1024004 & 5.0846170 \\
8.6926610 & 7.7412947 & 7.3972210 & 7.3825090 \\
\hline
\end{tabular}

always be positive provided that $m^{2}$ is not too negative. This leads to the consistent result on the QNMs of AdS soliton as disclosed in [14] that only normal modes exist as exhibited in table I and table II. Note that here the numerical results are shown in the case of $\lambda=k_{s}=0$.

This result indicates that the behavior of the perturbation of the scalar field coupling to Maxwell field does not change in the AdS soliton background with magnetic charged field, which is different from that in the charged AdS black hole. This property holds for higher dimensions. Unlike the charged AdS black hole that the perturbation will blow up when the black hole temperature is low enough or the coupling between scalar field and Maxwell field is strong enough, the perturbation in magnetic charged AdS soliton always keeps the normal modes. At the first sight, this is in contrary with the result reported in [33], where it was argued that like the AdS black hole case, there is holographic superconductor phase where the charged scalar condenses in the AdS soliton configuration. However in their discussion, they started with the neutral AdS soliton with the electric field where the electric potential is constant and the field strength is zero. In doing so $g^{t t}$ will appear in the effective mass term in their discussion. However in the magnetic charged AdS soliton background, the coordinate transformation forces $A=\Phi(r) d \phi_{s}$ to satisfy the Einstein equation and this brings $g^{\phi \phi}$ in the effective mass instead of the magic term $g^{t t}$ which can make the effective mass negative and lead to the drastic change in the behavior of the perturbation. Thus for the magnetic charged AdS soliton background, the consistent perturbation behavior tells us that there is no drastic phase change and the scalar field may not condensate in this configuration. This result is different from that of the neutral AdS soliton with the electric field. For the comparison we present numerical results of the perturbation in the neutral AdS soliton with the electric field in table III, where we find that when the electric potential is bigger, we do see that the 
TABLE III: Perturbations for the neutral AdS soliton with the electric field, we set $r_{+}=L=1, m^{2}=4$. We show the results of the first three modes with overtone numbers $n=0,1,2$ from the top to the bottom.

\begin{tabular}{|c|c|c|c|}
\hline$\Phi=0$ & $\Phi=2$ & $\Phi=3$ & $\Phi=4$ \\
\hline 2.7458368 & 1.8813878 & $1.2084619 i$ & $2.9086733 i$ \\
3.9089738 & 3.3585824 & 2.5060080 & $0.8484833 i$ \\
4.9738799 & 4.5540620 & 3.9673015 & 2.9562613 \\
\hline
\end{tabular}

quasinormal frequencies suddenly change from the purely real value to purely imaginary. The critical value of the electric potential to allow the sudden change of the quasinormal frequency decreases with the decrease of the mass of the scalar field, i.e. $\Phi_{\text {critical }}=1$ for $m^{2}=0, \Phi_{\text {critical }}=2$ for $m^{2}=1$ and $\Phi_{\text {critical }}=3$ for $m^{2}=4$. The positive imaginary frequency indicates that when the coupling between scalar and Maxwell field is strong enough, the AdS soliton with the electric field can become unstable and the superconducting phase can be formed.

\section{CONCLUSIONS AND DISCUSSIONS}

In this work we have studied the QNM of the perturbation of the scalar field interacting with the Maxwell field. Different from the single classical field perturbation, which always has the decay mode in the black hole background, we observed the possible growing mode when the perturbation of the scalar field strongly couples to the Maxwell field. This indicates that the interaction among classical fields can destroy the background spacetime. In the language of the AdS/CFT, this is due to the condensate of the scalar field on the black hole background and there is a second order transition between a non-superconducting state at high temperatures and a superconducting state at low temperatures. Our results disclose the signature of how this phase transition happens from the phenomenon of the perturbation. In the black hole background we have seen the influence of the topology and the dimensionality on the QNM when the scalar field is coupled with the Maxwell field.

We also discussed the perturbation in the AdS soliton background with magnetic field. We observed the consistent normal mode of the perturbation in the AdS soliton background with magnetic field and there is no characteristic of the phase transition in this configuration. This is different from that of the RN-AdS black hole background and indicates that possibly there is no scalar condensation in this AdS soliton configuration. In the discussion of [33], they started with the neutral spacetime with the electric field where the electric potential is constant and the field strength is zero. We have shown the signature of how the phase transition happens from the phenomenon of the perturbation in this background. It would be more interesting to investigate 
the electric charged AdS soliton background. For the AdS soliton background with electric charge, the exact solution of the Einstein equation can be found in the form

$$
d s^{2}=f d z^{2}+f^{-1} d r^{2}+\left(-r^{2} d t^{2}+r^{2} d y^{2}\right)
$$

where $f=-m / r-q^{2} / r^{2}+r^{2} / l^{2}$, and $A=q y d t$ is the gauge field. The appearance of the $y$ coordinate in the electric field makes the perturbation calculation much complicated. This solution itself is a new one and we will study this new soliton solution carefully in a separate future work.

Our investigation is still limited in the probe limit and has not scanned the full parameter space in the wave equations. Further work is needed to establish the full range of behaviors that the mechanism of the

perturbation exhibits. Besides it is also of interest to include the usual $|\Psi|^{4}$ term in the lagrangian and disclose its scalar perturbation dynamics, which will help get deeper understanding on the holographic condensation by comparing with the usual Ginzburg-Landau story. We look forward to reporting some of the progress on these issues in the future.

\section{Acknowledgments}

This work was partially supported by NNSF of China. We would like to acknowledge helpful discussions with Q. Y. Pan, S. Y. Yin, R. K. Su, S. F. Wu, X. H. Ge and Y. Q. Liu.

[1] H. P. Nollert, Class. Quant. Grav. 16, R159 (1999).

[2] K. D. Kokkotas and B. G. Schmidt, Living Rev. Rel. 2, 2 (1999).

[3] G. T. Horowitz and V. E. Hubeny, Phys. Rev. D 62, 024027 (2000).

[4] B. Wang, C. Y. Lin, and E. Abdalla, Phys. Lett. B 481, 79 (2000); B. Wang, C. Molina, and E. Abdalla, Phys. Rev. D 63, 084001 (2001); J.M. Zhu, B. Wang, and E. Abdalla, Phys. Rev. D 63, 124004 (2001); B. Wang, C. Y. Lin and C. Molina, Phys. Rev. D 70, 064025 (2004).

[5] V. Cardoso and J. P. S. Lemos, Phys. Rev. D 63, 124015 (2001); V. Cardoso and J. P. S. Lemos, Phys. Rev. D 64, 084017(2001); E. Berti and K. D. Kokkotas, Phys. Rev. D 67, 064020 (2003); V. Cardoso and J. P. S. Lemos, Class. Quantum Grav. 18, 5257 (2001); E. Winstanley, Phys. Rev. D 64, 104010 (2001); J. Crisstomo, S. Lepe and J. Saavedra, Class. Quant. Grav. 21, 2801 (2004); S. Lepe, F. Mendez, J. Saavedra, L. Vergara, Class. Quant. Grav. 20, 2417 (2003).

[6] D. Birmingham, I. Sachs, and S. N. Solodukhin, Phys. Rev. Lett. 88, 151301 (2002); D. Birmingham, Phys. Rev. D 64, 064024 (2001).

[7] B. Wang, E. Abdalla, and R. B. Mann, Phys. Rev. D 65, 084006 (2002); J. S. F. Chan and R.B. Mann, Phys. Rev. D 59, 064025 (1999); S. Musiri and G. Siopsis, Phys. Lett. B 576, 309 (2003); R. Aros, C. Martinez, R. Troncoso, and J. Zanelli, Phys. Rev. D 67, 044014 (2003); A. Nunez and A. O. Starinets, Phys. Rev. D 67, 124013 (2003).

[8] E. Abdalla, B. Wang, A. Lima-Santos, and W. G. Qiu, Phys. Lett. B 538, 435 (2002); E. Abdalla, K. H. CastelloBranco, and A. Lima-Santos, Phys. Rev. D 66, 104018 (2002).

[9] B. Wang, Braz. J. Phys. 35, 1029 (2005).

[10] S. Hod, Phys. Rev. Lett. 81, 4293 (1998); A. Corichi, Phys. Rev. D 67, 087502 (2003); L. Motl, Adv. Theor. Math. Phys. 6, 1135 (2003); L. Motl and A. Neitzke, Adv. Theor. Math. Phys. 7, 307 (2003); A. Maassen van den Brink, J. Math. Phys. 45, 327 (2004); O. Dreyer, Phys. Rev. Lett. 90, 08130 (2003); G. Kunstatter, Phys. Rev. Lett. 90, 161301 (2003); N. Andersson and C. J. Howls, Class. Quant. Grav. 21, 1623 (2004); V. Cardoso, J. Natario, and R. Schiappa, J. Math. Phys. 45, 4698 (2004); Jose Natario and Ricardo Schiappa, Adv. Theor. Math. Phys. 8, 
1001 (2004); V. Cardoso and J. P. S. Lemos, Phys. Rev. D 67, 084020 (2003); K. H. C. Castello-Branco and E. Abdalla, gr-qc/0309090.

[11] S. B. Chen, B. Wang and R. K. Su, Phys. Lett. B 647, 282 (2007).

[12] S. B. Chen, B. Wang and R. K. Su, gr-qc/0701088; E. Abdalla, R. A. Konoplya and C. Molina, Phys. Rev. D 72 , 084006 (2005); R. Konoplya, Phys. Rev. D 71, 024038 (2005); B. F. Schutz and C. M. Will, Astrophys. J. 291, L33 (1985); S. Iyer and C. M. Will, Phys. Rev. D 35, 3621 (1987); R. A. Konoplya, Phys. Rev. D 68, 024018 (2003); J. Y. Shen, B. Wang, and R. K. Su, Phys. Rev. D 74, 044036 (2006); E. Abdalla, B. Cuadros-Melgar, A. B. Pavan and C. Molina, Nucl. Phys. B 752, 40 (2006).

[13] G. Koutsoumbas, S. Musiri, E. Papantonopoulos, and G. Siopsis, JHEP 0610, 006 (2006).

[14] J. Shen, B. Wang, R. K. Su, C.Y. Lin, and R. G. Cai, JHEP 0707, 037 (2007).

[15] X. Rao, B. Wang, and G. H. Yang, Phys. Lett. B 649, 472 (2007).

[16] S. A. Hartnoll, C. P. Herzog, and G. T. Horowitz, Phys. Rev. Lett. 101, 031601 (2008).

[17] S. A. Hartnoll, C. P. Herzog, and G. T. Horowitz, J. High Energy Phys. 0812, 015 (2008).

[18] G. T. Horowitz and M. M. Roberts, Phys. Rev. D 78, 126008 (2008).

[19] E. Nakano and Wen-Yu Wen, Phys. Rev. D 78, 046004 (2008).

[20] I. Amado, M. Kaminski, and K. Landsteiner, J. High Energy Phys. 0905, 021 (2009); K. Maeda, M. Natsuume, and T. Okamura, Phys. Rev. D 79, 126004 (2009).

[21] G. Koutsoumbas, E. Papantonopoulos and G. Siopsis, J. High Energy Phys. 0907, 026 (2009).

[22] Q. Y. Pan, B. Wang, E. Papantonopoulos, J. Oliveira, and A. Pavan, arXiv: 0912.2475.

[23] J. Sonner, Phys. Rev. D 80, 084031 (2009); R.G. Cai and H. Zhang, arXiv: 0911.4867; R. A. Konoplya, A. Zhidenko, arXiv: 0909.2138.

[24] S. A. Hartnoll, arXiv: 0903.3246.

[25] C. P. Herzog, J. Phys. A 42, 343001 (2009).

[26] S. S. Gubser, arXiv: 0803.3483.

[27] S. S. Gubser and A. Nellore, JHEP 0904, 008 (2009).

[28] S. S. Gubser, Phys. Rev. D 78, 065034 (2008).

[29] F. Denef, S. A. Hartnoll, and S. Sachdev, arXiv: 0908.1788; S. A. Hartnoll, arXiv: 0909.3553.

[30] G. T. Horowitz and R. C. Myers, Phys. Rev. D 59, 026005 (1999)

[31] S. Surya, K. Schleich and D. M. Witt, Phys. Rev. Lett. 86, 5231 (2001)

[32] R. G. Cai, S. P. Kim, and B. Wang, Phys. Rev. D 76, 024011 (2007).

[33] T. Nishioka, S. Ryu, and T. Takayanagi, arXiv: 0911.0962.

[34] J. Y. Shen, B. Wang, and R. K. Su, Phys. Rev. D 74, 044036 (2006). 